\title{
UN ENFOQUE SOCIOECONÓMICO DE LA URBANIZACIÓN
}

\author{
Ricardo Cinta G. ${ }^{1}$ \\ El Colegio de México
}

\section{INTRODUCCIÓN}

Aunque existe una idea de la urbanización que se concibe como el crecimiento de las ciudades y como un complejo de cambios sociales y económicos aparejados con éste, no se ha logrado definir un concepto riguroso y operacional que sea útil para el análisis de estos cambios.

Sociólogos, economistas, planificadores, urbanistas y otros parecen coincidir en que este fenómeno, un tanto nebuloso en su concepción, es uno de los más representativos de las transformaciones estructurales que ocurren al desarrollarse un país. En el caso de México, se coincide en que es un fenómeno característico de la evolución del país en los últimos decenios.

$\mathrm{Si}$ bien es fácil definir conceptos operacionales y medibles de urbanización, como de cualquier otro fenómeno, es muy difícil hacerlo sin perder la perspectiva de su complejidad. Así, es fácil definir lo urbano en términos exclusivamente del número de habitantes: una comunidad es más urbana mientras más habitantes tenga. ${ }^{2} \mathrm{Si}$ bien los cambios sociales y económicos no son independientes de este crecimiento físico, es muy dudoso que el solo número de habitantes logre explicarlos en forma satisfactoria. El otro extremo está en las definiciones que intentan comprender un sinnúmero de aspectos del fenónemo, pero cuya amplitud no se presta para ningún uso analítico.

Ante estas circunstancias, cualquier intento de definición del fenómeno urbano como complejo social y económico se enfrenta a dificul-

1 El presente artículo está basado en el trabajo realizado por el autor como parte de un proyecto de investigación sobre la estructura y dinámica de la clase media mercantil en las ciudades pequeñas y medianas de México, dentro del programa de investigación del Centro de Estudios Económicos y Demográficos de El Colegio de México. En consecuencia, el material empírico y una parte de las consideraciones teóricas se refieren sólo a ciudades de mediano tamaño. Sin embargo, se pretende demostrar que el enfoque es válido en términos más generales. Este artículo condensa una parte, quizá la más importante, de los resultados presentados por el autor en su tesis profesional; ver referencia [3]. El autor agradece a Pedro Uribe, Jr., un sinnúmero de sugestiones y comentarios y la orientación matemático-stadística de este trabajo.

2 Sin embargo, aun a este nivel de simplicidad surgen problemas: la escala cuyas "marcas" están dadas por el número de habitantes ¿es una escala cardinal de urbanización o sólo lo es en el sentido ordinal? Ver más adelante un examen más amplio de este problema. 
tades. Por ejemplo, ¿coincide el fenómeno de "urbanización" con una mezcla de lo que los economistas conciben como desarrollo económico y lo que los sociólogos conciben como modernización, todo esto visto al nivel de una ciudad o de un conglomerado humano que habita en cierto lugar? ¿es el concepto de "urbanización", vía la idea de "vida urbana", una faceta del concepto de "bienestar"? Por el momento -y los resultados de este trabajo apuntan hacia una confirmación- estas interrogantes parecen no tener respuesta.

El enfoque de este trabajo es un poco distinto de un intento de definición del fenómeno urbano: se parte del supuesto de que, a medida que las ciudades crecen, se presentan cambios económicos y sociales, en parte explicados por el crecimiento y que a su vez lo explican en parte. El análogo estático de esta proposición es el siguiente: dada una colección de ciudades de distintos tamaños, existen características sociales y económicas diferentes entre ellas, que en parte son explicables por las diferencias de tamaños y en parte explican a éstas. El objeto de estudio de este trabajo es precisamente esta interrelación. Concretamente, se buscan dos objetivos:

a) analizar, mediante el análisis de varianza, las proporciones de explicación de las variaciones en el tamaño de las ciudades por medio de características sociales y económicas y de las variaciones de éstas por medio del tamaño de las ciudades;

b) definir un indicador del fenómeno urbano, o sea una función de variables sociales y económicas y del número de habitantes de una ciudad que represente en forma óptima las variaciones de sus componentes. ${ }^{3}$ Este indicador debe poseer propiedades cardinales, para ser analíticamente útil. Se le llamará "índice de urbanización".4

\section{LA INFORMACIÓN EMPLEADA: CARACTERÍSTICAS GENERALES}

El enfoque planteado en los párrafos anteriores se ha aplicado a datos sobre México. Fue preciso afrontar difíciles problemas de selección, tanto de variables (características económicas y sociales) como de unidades a ser estudiadas. En este inciso se describen estas últimas; más adelante se examina la selección de variables. Todos los datos se refieren al censo de población de 1960.

Desde luego, los datos pertinentes no existen al nivel de localidad, por lo que las unidades de estudio son municipios. Se ha limitado arbitrariamente el universo eliminando los municipios con localidades mayores de 50000 habitantes ${ }^{5}$ y los que contienen menos del $65 \%$ de su población en localidades de 5000 habitantes o más. Se piensa que los restantes son más o menos representativos de la vida propiamente urbana, sin llegar todavía a tratarse de grandes ciudades. En esta forma, la muestra ( $i$ ciertamente no aleatoria!) consiste en cien

3 En términos de varianza explicada, desde luego.

4 El vocablo puede ser impropio, y se admite que, ante las reflexiones hechas en párrafos anteriores, es ésta una licencia de lenguaje.

is Según el censo de 1960. 
municipios (en 1960 había 409 con localidades entre 5000 y 50000 habitantes); este grupo contiene 106 localidades entre 5000 y 50000 habitantes (en 1960 había 450 en este intervalo). Geográficamente, hay siete entidades federativas no cubiertas por la muestra, ${ }^{6}$ y ésta abarca 47 de las 111 zonas económicas de la Comisión Nacional de los Salarios Mínimos, utilizadas por Stern [22], [23], a las que se hará referencia más adelante. Finalmente, los municipios incluidos tienen una población total de 2139947 habitantes y las 106 localidades entre 5000 y 50000 habitantes absorben al $80 \%$ de esta población (1712538, según el censo de 1960).

Aunque no puede hablarse de una estratificación propiamente dicha, existe una clasificación, utilizada en el análisis de varianza: los municipios han sido agrupados en cinco clases, de acuerdo con la actividad principal de su población económicamente activa. Las clases son las siguientes:

Clase $A$ (agrícola-ganadera). El $65 \%$ o más de la población activa del municipio se dedica a la agricultura, ganadería, silvicultura, caza o pesca.

Las clases B, C, D y E se definieron tomando el resto de los municipios agrupados de acuerdo con la actividad que absorbe a la mayor parte de su población activa:

Clase $B$ (industrias de transformación)

Clase $C$ (industrias extractivas y de energía)

Clase $D$ (comercio)

Clase $E$ (servicios y transportes)

Para fines de análisis de varianza, se han considerado sólo cuatro intervalos de población. La distribución conjunta de los municipios por tamaño de población y "clase ocupacional" se resume en el cuadro 1.

\section{Cuadro 1}

Distribución de la MUESTRa POR NÚMERO dE habITANTES Y ClaSe OCUPACIONAL

\begin{tabular}{crrrrrr}
$\begin{array}{c}\text { Intervalo } \\
\text { de } \\
\text { población }\end{array}$ & Clase & & & & & \\
& $A$ & $B$ & $C$ & $D$ & $E$ & Suma \\
\hline $5000-14999$ & 38 & 3 & 3 & 2 & 2 & 48 \\
$15000-29999$ & 12 & 4 & 3 & 0 & 10 & 29 \\
$30000-49999$ & 2 & 2 & 1 & 2 & 7 & 14 \\
50000 en & 1 & 1 & 1 & 2 & 4 & 9 \\
adelante & 1 & 10 & 8 & 6 & 23 & 100 \\
$\quad$ Suma & 53 & & & \\
\hline
\end{tabular}

6 Distrito Federal, Tamaulipas, Durango, Querétaro, Tabasco, Sinaloa y Aguascalientes. 


\section{LA TEORIA SOCIOLÓGICA Y LA SELECCIÓN DE VARIABLES}

Evidentemente, un análisis de este tipo tiene mayor probabilidad de éxito mientras más información utilice, tanto en el sentido de un tamaño aceptable de muestra y en el de un número satisfactorio de variables, como en el de un máximo de información a priori dada por la teoría sociológica de la urbanización. Pero el investigador tropieza con escollos bastante serios: la muestra escogida aquí es relativamente pequeña, aun cuando puede considerarse como universo puesto que incluye a todos los municipios que cumplen con las condiciones de "pequeña y mediana urbanización" definidas en la introducción. Por otra parte, las variables a ser analizadas han de escogerse de entre aquellas sobre las que existe información censal ${ }^{7}$ a nivel municipal $; 8$ por desgracia, su número no es muy elevado. Sin embargo, el escollo más importante estriba en que la sociología moderna no ha producido todavía una teoría aceptable del fenómeno urbano. Con todo, cabe señalar los elementos principales que puede aportar la teoría sociológica existente, a partir de Weber, para esclarecer un poco el campo de investigación.

El punto de partida de un análisis socioeconómico del fenómeno urbano, a la vez que para el abandono de un criterio de "aglomeración de habitantes" para definirlo, parece ser el señalamiento de las funciones económicas de la ciudad, concretamente de la diversificación de actividades y de la creación de un mercado, que hace Weber ([28], Vol. II, pp. 938-939) para definir el carácter urbano de un asentamiento permanente de población.

Sin embargo, es inmediato pensar en elementos sociales, políticos, culturales, etc., que pueden llegar a definir lo que Wirth [30] llama el "modo de vida" urbano. Lo interesante es que el "modo de vida" urbano ha sido objeto de un sinnúmero de explicaciones y definiciones circulares; por ejemplo, Dorselaer y Gregory ([8], p. 12) definen "la urbanización en un sentido sociológico ...[como]... el pasaje o mutación de un estilo de vida rural a un estilo de vida urbana: en otros términos, un cambio gradual de una sociedad de tipo rural a una sociedad de tipo urbano", aclaración con la cual el lector permanece exactamente donde se encontraba. Algo más añade Wirth al considerar el volumen, la densidad y la heterogeneidad de los individuos que constituyen la aglomeración humana como elementos distintivos de un núcleo urbano.

Hay una idea interesante planteada por Redfield y Singer [18] y que resulta ser sumamente útil en el análisis socioeconómico del fenómeno urbano; estos autores consideran una clasificación dual del desarrollo de las ciudades: por un lado, hablan de la "urbanización primaria" de cambio "ortogenético" que no niega ni destruye la vieja cultura, sino que la desarrolla, dentro del mismo sistema de valores, dándole una expresión "culta". Por otro lado, la "urbanización secundaria", de cambio "heterogenético", que sustituye la vieja cultura y

7 Incluyendo censos de población, industria, comercio y servicios y agrícolaganadero.

8 Puesto que no la hay al nivel de localidades. 
los viejos valores por un sistema en que lo racional, lo técnico, el predominio de relaciones secundarias, el individualismo, etc., definen una nueva forma de vida (véase en Germani [10] una excelente caracterización). Es tentador asociar la "urbanización secundaria" a procesos de desarrollo económico repentino y rápido, sobre todo a procesos de industrialización; la "urbanización primaria" existe ya cuando la civilización industrial aparece.

No resulta entonces extraño que Hoselitz ([13], p. 146) al hacer consideraciones de carácter bastante general, y Bazzanella ([1], p. 18) al tratar de explicar el desarrollo urbano de Brasil, se muestran profundamente insatisfechos de la identificación de los procesos de crecimiento urbano e industrialización como posible explicación del primero, a que conduciría la adopción sin restricciones de la ya mencionada caracterización weberiana. Las ciudades sujetas a "urbanización primaria" sufren lo que los sociólogos actuales han llamado "modernización". Siguiendo a Véliz [27], se afirmaría que la industrialización, que llegó a buena parte de los países latinoamericanos como "accidente histórico", encontró una civilización preindustrial urbana de mayorías alfabetas y de minorías suficientemente preparadas para absorber la tecnología industrial; sus características sociales son entonces muy distintas de las europeas. Recientemente se ha propuesto una hipótesis que pretende conciliar, al menos en parte, la caracterización weberiana con los conceptos de "urbanización primaria", "urbanización preindustrial", "modernización", etc., y las explicaciones que se derivan de ellos. Se afirma que el crecimiento de los llamados "sectores terciarios" (comercio, servicios y transportes) está asociado en tal forma al paso de una agricultura de subsistencia a una agricultura de mercado, que la transformación inmediata de una sociedad rural hacia el "modo de vida urbana" encuentra una explicación mucho más viable en la transición de su actividad económica de lo agrícola a lo "terciario" que de lo agrícola a lo industrial. En esta forma, las sociedades, desarrolladas por cambios "ortogenéticos", en el lenguaje de Redfield y Singer, se llegan a transformar en urbanas antes de su industrialización. Esto se opone un tanto a la llamada "teoría de los tres sectores", de Colin Clark [4] y J. Fourastié [9], empíricamente sostenible con evidencia europea, pero no confirmable con material empírico latinoamericano: en Clark y Fourastié la transición es agrícola-industrial-"terciaria" (véase en Dorselaer [7] un análisis detallado).

Esta es la "hipótesis de terciarización", sugerida principalmente por Dorselaer [7], Bazzanella [1] y Germani [10], [11], entre otros, y que es en cierta forma la hipótesis subyacente en el proyecto al que pertenece este trabajo, ${ }^{9}$ cuando menos respecto al crecimiento de las ciudades pequeñas y medianas de México.

La hipótesis de "terciarización" tiene implicaciones importantes en la sociología del desarrollo. Si bien es posible que las sociedades agrícolas se transformen en urbanas preindustriales a la vez que sufran cambios estructurales, como la introducción de regímenes de seguridad colectiva, la elevación de los niveles educativos y otros seme- 
jantes, la transición vía el crecimiento de los sectores comerciales y de servicios puede significar la persistencia de formas arcaicas de organización social, dada la carencia de los elementos de presión hacia el cambio, normalmente asociados al surgimiento de un proletariado industrial. Esto podría explicar la presencia de estructuras tradicionales al lado de elementos "modernos", característica común del "dualismo" del desarrollo latinoamericano, por ejemplo. Sin embargo, estas consideraciones son materia de otras investigaciones, y se mencionan como una de las muchas razones por las que la comprobación de la hipótesis adquiere particular importancia en el contexto de la sociología urbana así como de la sociología del desarrollo latinoamericano.

Recientemente, Reyna, Villa y Albrechtsen [19] han encontrado una relación interesante enitre el proceso de "terciarización" y la estratificación ocupacional en México. Vale la pena, entonces, explorar la relación entre la estratificación ocupacional y el fenómeno urbano. En este trabajo se hacen exploraciones preliminares a este respecto.

Las consideraciones de los párrafos anteriores parecen apuntar hacia cuatro grandes grupos de elementos a explorar como condicionantes de la "vida urbana".

I. Estructura económica. Analizable a través de la estructura de la producción y de la ocupación. Los indicadores disponibles apuntan hacia la conveniencia de estudiar, sobre todo, composición sectorial. Existen además datos sobre el número de establecimientos en la industria, el comercio y los servicios.

II. Estructura social. Estudiable a través de indicadores que reflejen estratificación - al menos ocupacional- y predominio de relaciones secundarias; el único indicador del segundo tipo derivable de material censal es un indicador de "burocratización", basado en la proporción de población asalariada.

III. Estructura cultural. Definida por indicadores de tipo educativo. No existe material censal fuera de datos sobre alfabetismo y escolaridad.

IV. Facilidades y comodidades "urbanas". Estudiables sólo a través de indicadores referentes a las condiciones de habitación y servicios conexos.

Las siguientes son las variables seleccionadas, dentro de cada grupo:

I. Estructura económica :

$X_{1}$ : valor de la producción agrícola

$X_{2}$ : valor de la producción industrial

$X_{3}$ : número de establecimientos industriales

$X_{4}$ : número de establecimientos comerciales

$X_{5}$ : número de establecimientos de servicios

$X_{6}$ : proporción no agrícola de la población económicamente activa

II. Estructura social :

$X_{7}$ : burocratización (proporción asalariada de la población económicamente activa) 
$X_{8}$ : estratificación (relación del número de trabajadores manuales al número de trabajadores no manuales) ${ }^{10}$

\section{Estructura cultural}

$X_{9}$ : alfabetismo (proporción alfabeta de la población mayor de 6 años)

$X_{10}$ : escolaridad infantil (promedio de años cursados por la población de 7 a 14 años)

$X_{11}$ : escolaridad juvenil (promedio de años cursados por la población de 15 a 29 años)

IV. Facilidades y comodidades "urbanas":

$X_{12}$ : proporción de viviendas con servicio de agua entubada (dentro del edificio).

$\mathrm{X}_{13}$ : hacinamiento (indicador "inverso"; definido como el número de habitantes por cuarto, cifra media municipal).

Finalmente, se ha incluido.

$\overrightarrow{\mathrm{X}}_{14}$ : número de habitantes en el municipio

Los párrafos próximos se consagran al análisis empírico de estas variables; el apartado 4 trata sobre la explicación de las variaciones de los indicadores escogidos vía el tamaño del municipio y vía su actividad económica predominante.

\section{ANÁlisis de la varianZa de las CaRacterísticas uRbanas}

Se ha planteado la hipótesis de que la actividad predominante tiene mayor poder de explicación de las variaciones en las "características urbanas" $X_{1}, X_{2}, \ldots, X_{13}$ que el tamaño del municipio. Acudiendo a la clasificación presentada en la introducción, se ha hecho el análisis de varianza en un sentido, para la clasificación por tamaños y para la clasificación por clases ocupacionales. Los resultados se resumen en el cuadro 2 . Se advierte de inmediato gue a pesar de que en la mayor parte de los casos tanto las diferencias entre clases como las diferencias entre tamaños son significativas al nivel del $1 \%$, la proporción de varianza entre clases es mayor que la proporción de varianza entre tamaños en nueve de las trece variables analizadas. Aunque cada "explicación" (clase vs. tamaño) tiene un caso en que las diferencias no son significativas al $5 \%$, la clase ocupacional presenta diferencias significativas al $1 \%$ en el resto, salvo una de las variables (burocratización) significativas al $5 \%$. El tamaño implica diferencias significativas al $1 \%$ en sólo ocho de las trece variables. Parece, entonces, que en estos términos muy generales, la evidencia no contradice la hipótesis de mayor poder explicativo de la ocupación que del tamaño.

Hay algunas observaciones interesantes que hacer: si bien las diferencias entre clases son significativas al nivel del $1 \%$ para las variables de tipo "cultural", parece que es la clase agrícola la que deter-

10 Variable sugerida por R. Stavenhagen. Se analizó, pero no fue incluida en el "índice de urbanización". 


\section{Cuadro 2}

Proporciones DE INTRAVARIANZAS A VARIANZA TOTAL

\begin{tabular}{lcc}
\hline \multicolumn{1}{c}{ Variable } & Entre tamaños & Entre clases \\
\hline 1. Producción agrfcola & $.441 \pm$ & $.155 \pm+$ \\
2. Producción industrial & $.325 \pm$ & .553 \\
3. Establecimientos industriales & .910 & .479 \\
4. Establecimientos conerciales & .875 & .398 \\
5. Establecimientos de servicios & .767 & .390 \\
6. Mano de obra no agricola & .509 & .911 \\
7. Burocratizacion & .877 & $.399 \pm$ \\
8. Estratificacion & .595 & .829 \\
9. Alfabetismo & $.382 \pm$ & .661 \\
10. Escolariaad infantil & $.459 \pm$ & .630 \\
11. Escolaridad juvenil & $.410 \pm$ & .740 \\
12. Servicios de agua & .608 & .602 \\
13. Hacinamiento & $.164 \pm+$ & .571 \\
\hline
\end{tabular}

* Significativa al $5 \%$.

** No significativa al $5 \%$.

Sin indicación: Significativa al $1 \%$.

mina por completo estas diferencias; en efecto, el alfabetismo presenta medias no significativamente distintas entre clases no agrícolas, al igual que el nivel educativo de 15 a 29 años. Para la educación infantil, parecen distinguirse tres grupos: la clase agrícola, con una media de 1.43 años cursados, las clases industrial de transformación y comercial, con medias de 1.91 y 1.95 años, no significativamente distintas al nivel del $5 \%, 11$ y el resto de las clases industriales y las de servicios y transportes, con medias de 2.05 y 2.13 años, tampoco significalvamente distintas al $5 \%$.

Otro caso interesante es el del "hacinamiento": de nuevo, las diferencias entre clases son significativas al nivel del $1 \%$, pero las diferencias entre clases no agrícolas no lo son al $5 \%$. Se advierte, sin embargo, una media de hacinamiento ligeramente menor en los municipios terciarios que en los secundarios.

Dados estos resultados, surge la siguiente pregunta: ¿hasta qué grado puede la actividad predominante explicar el tamaño del municipio? En este terreno se cuenta además con datos al nivel de localidades. El cuadro 3 resume los datos al nivel de localidades junto con los de carácter municipal. 
Cuadro 3

DisTRIbUCIÓN DE LA POBLACIÓN, LOS MUNICIPIOS Y LAS LOCALIDADES POR CLASES OCUPACIONALES

\begin{tabular}{|c|c|c|c|c|c|c|}
\hline Clase & $\begin{array}{c}1 \\
(\operatorname{Ag} \times 1 \operatorname{cola})\end{array}$ & $\begin{array}{c}B \\
\text { (Industria } \\
\text { de trane } \\
\text { formacion) }\end{array}$ & $\begin{array}{c}c \\
\text { (otras in } \\
\text { dustrias) }\end{array}$ & $\begin{array}{c}D \\
\text { (Comercio) }\end{array}$ & $\begin{array}{c}\mathrm{E} \\
\text { (Servicios } \\
y \text { transpor } \\
\text { tes) }\end{array}$ & Total \\
\hline $\begin{array}{l}N^{\circ} \text { de } \\
\text { localidades }\end{array}$ & 54 & 12 & 10 & 6 & 24 & 106 \\
\hline $\begin{array}{l}N^{\circ} \text { de } \\
\text { municipios }\end{array}$ & 53 & 10 & 8 & 6 & 23 & 100 \\
\hline $\begin{array}{l}\text { Tamaño medio } \\
\text { de la locali- } \\
\text { dad } y\end{array}$ & 10.26 & 10.76 & .17 .00 & 28.29 & 25.74 & 16.16 \\
\hline $\begin{array}{l}\text { Tamaño medio. } \\
\text { del maniei- } \\
\text { pto } 1\end{array}$ & 13.60 & 13.97 & 26.38 & 36.10 & 32.51 & 20.36 \\
\hline $\begin{array}{l}\text { Varianza del } \\
\text { tamaño do lo- } \\
\text { calidad } 2 /\end{array}$ & 42.90 & 23.47 & 85.24 & 230.94 & 156.37 & 127.98 \\
\hline $\begin{array}{l}\text { Varianza del } \\
\text { tamaño del. } \\
\text { municipio } 2 /\end{array}$ & 78.94 & 183.93 & 357.11 & 413.51 & 53.98 & 206.03 \\
\hline $\begin{array}{l}\text { Proporcion de } \\
\text { la población } \\
\text { de las looali- } \\
\text { dades }\end{array}$ & 32.35 & 11.75 & 9.92 & 9.90 & 36.08 & 100.00 \\
\hline $\begin{array}{l}\text { Proporcion de } \\
\text { la poblacion } \\
\text { municipal }\end{array}$ & 33.68 & 11.33 & 9.86 & 10.12 & 34.96 & 100.00 \\
\hline
\end{tabular}

1. Miles de habitantes.

2 Millones de habitantes.

Al nivel de localidad, la varianza del tamaño entre clases es el $36.9 \%$ de la varianza total, de donde las diferencias entre clases son significativas al $5 \%$; al nivel de municipios la proporción es del $38.8 \%$ y las diferencias son significativas al nivel del $1 \%$.

Estos argumentos confirman la idea de una determinación del tamaño de las ciudades y de sus características "urbanas", a través de la estructura de su población activa, que refleja, en parte, la posible validez de la caracterización weberiana del fenómeno urbano.

Finalmente, es interesante explorar la dependencia o independencia del crecimiento de las localidades, respecto a su tamaño inicial. Las implicaciones del resultado son interesantes. En primer lugar, es fácil ver que si la tasa de crecimiento resulta independiente del tamaño inicial, la distribución del tamaño tiende a la log-normal (véase en [21] una demostración). Por otra parte, si resulta que el crecimiento es explicado por un modelo log-normal, como el producto de incrementos aleatorios independientes en el tiempo, el sociólogo debe bus- 
car explicaciones del fenómeno urbano distintas a la del tamaño de las ciudades; el efecto acumulado de causas sociales, económicas, políticas, etc., se presenta en la forma de incrementos aleatorios independientes.

En este caso se ha encontrado que, efectivamente, las tasas de crecimiento de 1950 a 1960 de las 106 localidades son independientes del tamaño que tenían éstas en 1950. Por otra parte, la distribución del tamaño de las localidades no se desvía demasiado de una log-normal truncada en 5000 y 50000 habitantes. Desde luego, esta distribución empírica es también compatible con una distribución de Pareto para la cola de más de $\mathbf{5 0 0 0}$ habitantes. Esto apunta hacia la necesidad de estudiar más a fondo el proceso estocástico de crecimiento de las ciudades, tanto en la teoría como en lo empírico.

\section{SOBRE LA CONSTRUCCIÓN DE UN ÍNDICE DE URBANIZACIÓN}

El objeto de esta sección es presentar la construcción de un indicador que sintetice las variables que aquí se han llamado "características urbanas"; será llamado "índice de urbanización".

La construcción de un índice de este tipo plantea problemas teóricos (sociológicos y urbanísticos) y estadísticos bastante interesantes. Desde luego, las consideraciones del apartado 3 llevan a descartar indicadores basados solamente en el número de habitantes. Entre ellos se encuentran los difundidos "índices" de Davis y Casis [5] y Davis y Golden [6] que, además, no implican ningún razonamiento medianamente elaborado sobre el fenómeno urbano.

Independientemente de que estos "índices" difieren en objetivo de lo que aquí se intenta elaborar, apuntan a una característica fundamental a ser buscada y que no poseen: los "índices" mencionados no poseen propiedades cardinales. Si bien con el "índice" de Davis y Golden es posible afirmar si un conglomerado es "más urbano" que otro (de acuerdo con el número de habitantes), el sistema arbitrario de ponderación de intervalos de población que usa no permite afirmar qué tanto más urbano es el primero que el segundo. Aquí se apunta una contradicción en el razonamiento de sus autores: el método de ponderación se justifica diciendo que no hay proporcionalidad en el incremento de "lo urbano" y el incremento del número de habitantes, o sea que se descarta el número de habitantes por ser un indicador ordinal y no cardinal. Pero si ese algo nebuloso "lo urbano" crece con el cuadrado, o la exponencial o alguna otra función del número de habitantes, no queda claro nunca. La arbitrariedad del sistema de ponderación hace caer de nuevo (si es que se cae en algo) en un indicador ordinal. En la elaboración de un índice de urbanización se trata de evitar este tipo de razonamientos poco rigurosos.

La carencia de una teoría adecuada, que aporte las bases -a la manera en que la teoría de la demanda las aporta para los índices de precios- para ponderar las variables que definen "lo urbano", implica la necesidad de consideraciones extrasociológicas. Hasta ahora sólo se han producido índices con pesos constantes, o sea índices en los que el peso de cada variable es el mismo, cualquiera que sea la ciudad, división geográfica, período u otro tipo de observación a que 
se aplique. ${ }^{12}$ Es probable que una formulación teórica adecuada permita, como en la teoría económica, llegar a un índice con pesos variables; las implicaciones sociológicas de esto son en extremo interesantes, pero no serán examinadas aquí.

Entre los índices que utilizan más de una variable, y para los que no existe base teórica de ponderación, sólo se han producido índices lineales. Si bien en la teoría económica se han justificado tanto índices lineales (a través del trabajo de Konyus) como índices geométricos (a través del trabajo de Theil) como aproximaciones locales a un índice cardinalmente perfecto (de utilidad constante), no es posible señalar la forma óptima para un índice de urbanización, de educación, etc., a menos que exista una teoría que lo permita. Sin embargo, un índice lineal es una aproximación local aceptable a cualquier función que resulte ser óptima, en una vecindad suficientemente pequeña.

Por otra parte, no existe una teoría para índices no lineales sin bases teóricas de ponderación, 13 por lo que por el momento debe acudirse a índices lineales, cuyas propiedades cardinales son válidas sólo en el sentido de una aproximación local.

Ha habido intentos - las obras que los contienen han sido bastante difundidas- de elaborar índices lineales en terrenos no económicos. Pero su construcción no ha resultado de ningún razonamiento elaborado $\mathrm{y}$, estadísticamente, resultan ser trabajos de aficionado. Como ejemplos claros están el "índice de educación" de Harbison y Myers [12] y el "índice de pobreza" de Wilkie [29]. Se trata de promedios aritméticos no ponderados de números relativos, de variables escogidas tras de algún razonamiento no elemental.

En el terreno económico hay intentos bastante más fructíferos: Carter, Reddaway y Stone [2] han aplicado la técnica de los componentes principales del análisis multivariado para obtener un índice de producción, Tintner [26] a índices de precios, etc. Por otra parte, Theil [24] [25], Kloek y de Wit [16] y Kloek y van Rees [17] han desarrollado la teoría de los "índices lineales óptimos" de precios y cantidades.

En este trabajo se ha aplicado la técnica de los componentes principales para obtener un índice a partir de las variables definidas en la sección 3 como "características urbanas", omitiendo la variable de estratificación (incluida en el análisis después del cálculo del índice) y agregando el número de habitantes del municipio.

Vale la pena explicar, en términos muy breves, la naturaleza del método de componentes principales y su interpretación en términos del índice de urbanización. Las observaciones sobre las trece variables en los cien municipios pueden representarse geométricamente como un conglomerado de cien puntos en espacio de dimensión trece. El orden de variación de las trece variables puede medirse como el volumen del menor paralelepípedo de dimensión trece que contenga todos estos puntos. La figura 1 ilustra esta idea para el caso de dos variables:

12 Los índices usados comúnmente para precios y cantidades en economía tienen pesos variables.

13 En el Centro de Estudios Económicos y Demográficos de El Colegio de México, Pedro Uribe, Jr., trabaja actualmente en la teoría de los índices "geomé. tricos óptimos". 
cada punto corresponde a un municipio; sus coordenadas son dos "características urbanas", $X_{1}$ y $X_{2}$. El orden de variación de la muestra está dado por el área del rectángulo $R$, que contiene a todos los puntos-muestra. Estadísticamente, este "orden de variación" corresponde al concepto de "varianza generalizada", que se define como el determinante de la matriz de dispersión de la muestra.

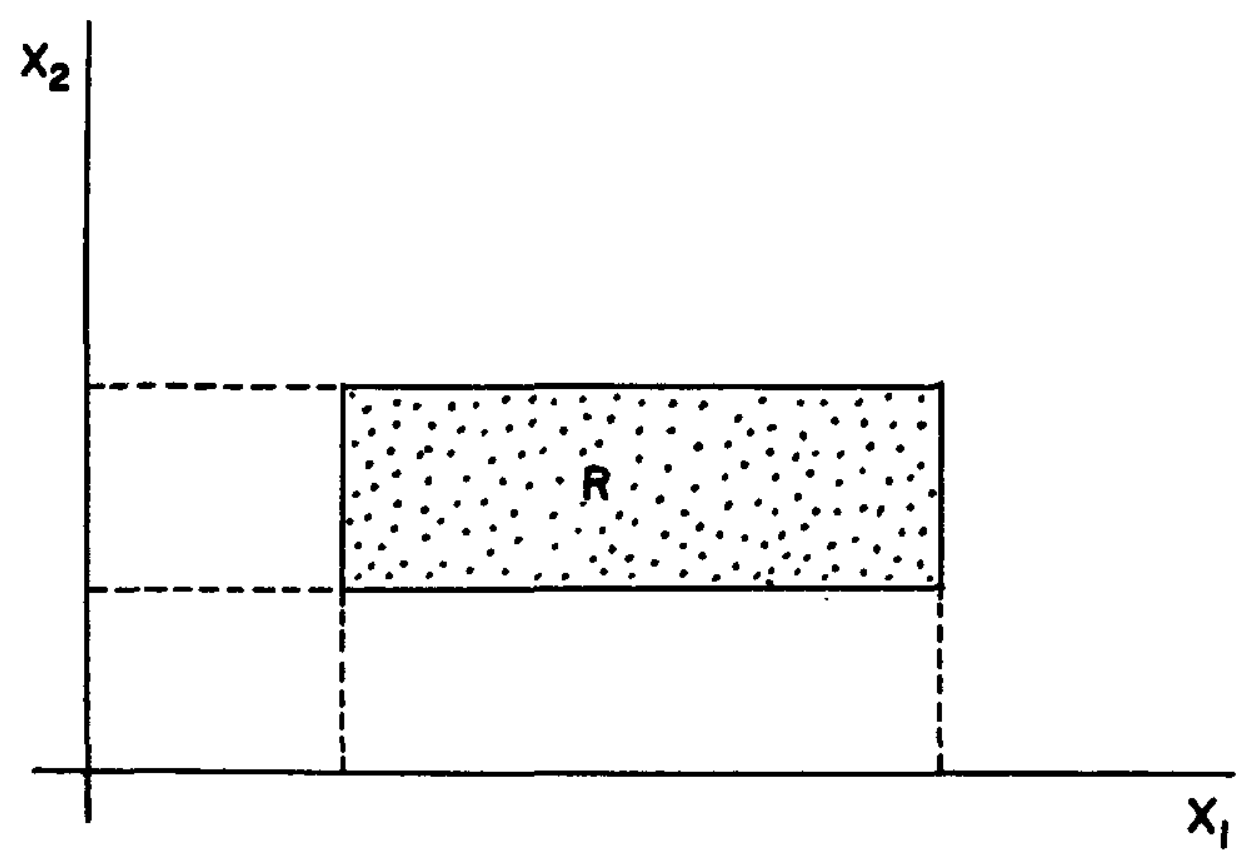

Figura I

El problema a resolver es el siguiente: encontrar un sistema de coordenadas, funciones lineales de las variables originales y dado por ejes perpendiculares, tal que: i) la relación de la varianza de la primera coordenada a la varianza generalizada de la muestra sea un máximo, y ii) la relación de la varianza de la segunda coordenada a la varianza generalizada sea un máximo del residuo "no explicado" por la primera coordenada, etc.

Inmediatamente se ve que la primera coordenada de este nuevo sistema, tomada como indicador, tiene un máximo poder de explicación (medido como relación entre varianzas) del fenómeno multidimensional representado. Hotelling [15] llama a las nuevas coordenadas los componentes principales de la muestra, y a sus varianzas los componentes principales de la varianza generalizada.

La obtención de los componentes principales es bastante simple: cada uno de ellos es función lineal de las variables originales; los coeficientes de estas funciones son elementos de los vectores característicos de la matriz de dispersión, ordenados en forma de que el primer componente principal tenga como coeficiente los elementos del vector característico asociado a la máxima raíz característica de la matriz de dispersión, el segundo corresponda en igual forma a la segunda. mayor raíz característica, etc.

Hotelling mismo [16] ha demostrado que los componentes principales se pueden obtener sustituyendo la matriz de dispersión por la matriz de correlación de las variables originales. En tal caso, la "re- 


\section{Cuadro 4}

Matriz de correlación de las "Características URbanas"a

\begin{tabular}{|c|c|c|c|c|c|c|c|c|c|c|c|c|c|}
\hline$i^{j}$ & 1 & 2 & 3 & 4 & 5 & 6 & 7 & 8 & 9 & 10 & 11 & 12 & 13 \\
\hline 1 & 1 & .8298 & .8120 & .7718 & .9235 & .6046 & .3020 & . 3499 & .0107 & .1944 & -.1178 & -.6360 & -.6790 \\
\hline 2 & & 1 & .9415 & .6751 & .3105 & .5160 & .3128 & .0485 & .0289 & .2172 & -.0451 & -.4199 & -.7127 \\
\hline 3 & & & 1 & .5917 & .8484 & .3724 & .2246 & .3516 & .0255 & .2104 & -.0524 & -.4400 & -.5140 \\
\hline 4 & & & & 1 & .6434 & $\cdot 5920$ & .4256 & .4253 & .1512 & .1508 & .0091 & -.5714 & -.0636 \\
\hline 5 & & & & & 1 & $\cdot 3557$ & .0937 & .4121 & -.0374 & . 1994 & -.1459 & -.6329 & -.5531 \\
\hline 6 & & & & & & 1 & .4351 & .0974 & .0579 & .0393 & -.0593 & -.4103 & -.3999 \\
\hline 7 & & & & & & & 1 & .5518 & .7829 & .0569 & .7351 & .2279 & -.9374 \\
\hline 8 & & & & & & & & 1 & .7726 & .0338 & .0952 & -.1531 & -.5790 \\
\hline 9 & & & & & & & & & 1 & .0095 & .9732 & .3370 & -.3231 \\
\hline 10 & & & & & & & & & & 1 & -.0030 & -.1044 & -.1577 \\
\hline 11 & & & & & & & & & & & 1 & .5079 & -.2521 \\
\hline 12 & & & & & & & & & & & & 1 & .4172 \\
\hline 13 & & & & & & & & & & & & & 1 \\
\hline
\end{tabular}

a El orden de las variables es el dado al final de la sección $3 ; \mathrm{X}_{8}$ es aquí no "estratificación", sino el número de habitantes del municipio. 
presentatividad" o "poder de explicación" del componente principal número $i$ en orden, está dada por:

$$
r_{i}=\frac{\lambda_{i}}{n}
$$

en que $\lambda_{i}$ es la raíz característica número $i$ en orden de magnitud de la matriz de correlación, y $n$ es el número de variables originales.

En este trabajo, el índice de urbanización está dado por el primer componente principal de la muestra de las variables $X_{1}$ a $X_{7}, X_{9}$ a $X_{13}$ y el número de habitantes, definidas en la sección 3. La sección 6 analiza los resultados empíricos a este respecto.

\section{El INDICE DE URBANIZACIÓN DE LOS CIEN MUNICIPIOS}

El cuadro 4 es la matriz de correlación de las trece variables, en que $X_{8}$ es ahora el número de habitantes del municipio. En vista de que el primer vector característico de esta matriz no es positivo, no se ha adoptado ningún procedimiento de normalización. La suma de los pesos del índice es entonces 2.1338, aproximadamente. El cuadro 5 presenta los pesos de cada una de las variables, reordenadas según su posición en el índice. Se advierte que aquí se han incluido dos varia-

\section{Cuadro 5}

Pesos de las variables EN EL ÍNDICE DE URBanización

\begin{tabular}{lll}
\hline Variable Núm. de & Definicion & Coeficiente
\end{tabular}

\begin{tabular}{lrll}
\hline$x_{6}$ & 1 & Poblacion activa no agricola & .3808 \\
$x_{9}$ & 2 & Alfabetismo & .3535 \\
$x_{10}$ & 3 & Escolaridad infantil & .3522 \\
$x_{12}$ & 4 & Viviendas con agua entubada & .3506 \\
$x_{7}$ & 5 & Burocratizacion & .3498 \\
$x_{8}$ & 6 & Población municipal & .2530 \\
$x_{5}$ & 7 & Establecimientos de servicios & .2195 \\
$x_{2}$ & 8 & Produccion industrial & .2166 \\
$x_{4}$ & 9 & Establecimientos comerciales & .1011 \\
$x_{11}$ & 10 & Escolaridad juvenil & .0987 \\
$x_{3}$ & 11 & Establecimientos industriales & .0471 \\
$x_{1}$ & 12 & Produccion agricola & -.2403 \\
$x_{13}$ & 13 & Hacinamiento & -.3488 \\
\hline
\end{tabular}


bles "anti-urbanas", o sea, dos indicadores que varían inversamente con el índice: el valor de la producción agrícola y el "hacinamiento", cuyos coeficientes en el índice son negativos. La negatividad del último llama un poco la atención, puesto que se conjetura que a medida que las ciudades se desarrollan, las condiciones de vida de sus habitantes implican una mayor "aglomeración" en la vivienda. Se observa que el coeficiente de correlación del hacinamiento es positivo sólo con la producción agrícola; con el tamaño es negativo, pero no muy grande en valor absoluto ( -.58 ), menor (también en valor absoluto) que con alfabetismo (-.71) o establecimientos de servicios (-.84).

El vector de coordenadas (en el orden original de las variables) iguales a los coeficientes en el cuadro 5 corresponde a la raíz característica $\lambda_{1} \cong 5.9479$, la máxima de la matriz de correlación (cuadro 4). Se tiene entonces que la representatividad del índice está dada por:

$$
r_{1}=\frac{5.9479}{13} \cong .4575
$$

o sea, de un $46 \%$ aproximadamente, valor que no puede considerarse muy elevado. Probablemente, la inclusión de variables como el ingreso per capita y algún indicador de su desigualdad (Pareto, Gini, medidas de información), junto con indicadores de estratificación, de movilidad ocupacional, participación política, etc., llevaría tanto a una mayor representatividad de un índice como a un concepto más adecuado de "urbanización".

Definidas numéricamente las trece variables como proporciones de su media de muestra, el índice de urbanización ha sido calculado para los cien municipios. Si bien el "municipio medio", o sea aquel en el que todas las variables adoptan como valor su media de muestra, presentaría el valor 2.1338 para el índice de urbanización, la media de éste sobre los cien municipios es 1.8991 . La varianza es 1.5453 , o sea un $65 \%$ de la media. Observando por clases ocupacionales, se aprecia una relación bastante estrecha entre el nivel del índice y la actividad predominante en los municipios : no existen municipios agrícolas con un índice elevado de urbanización ni no agrícolas con niveles bajos ( salvo uno de actividad industrial extractiva y otro de actividad comercial: San Pablo del Monte, Tlaxcala, y Allende, Coahuila, respectivamente).

El análisis de varianza del índice es interesante; el cuadro 6 condensa la distribución del índice por clases ocupacionales y las medias de clase. Se obtiene una varianza entre clases de .8252 , que es el $53.4 \%$ de la varianza total; las medias de clase son distintas con nivel de significación del 1\%; sin embargo, las medias de las clases no agrícolas no son significativamente distintas al $5 \%$.

Con el tamaño sucede algo interesante: las medias del índice en los cuatro intervalos de tamaño son significativamente distintas al $1 \%$; la varianza entre tamaños es un $57.15 \%$ de la varianza total. Ahora bien, los dos primeros intervalos de tamaño ( 5 a 15000 habitantes y 15 a 30000 ) tienen medias de urbanización significativamente distintas al nivel de $1 \%$; los dos intervalos superiores no difieren al $5 \%$. 


\section{Cuadro 6}

DISTRIBUCIÓN DEL ÍNDICE DE URBANIZACIÓN POR CLASES OCUPACIONALES

\begin{tabular}{|c|c|c|c|c|c|}
\hline $\begin{array}{l}\text { Valor } \\
\text { del } \\
\text { Indice }\end{array}$ & $\begin{array}{c}A \\
\text { (Asrícola) }\end{array}$ & $\begin{array}{c}\text { B } \\
\text { (Industria } \\
\text { de trans } \\
\text { formación) }\end{array}$ & $\begin{array}{c}\text { c } \\
\text { (otras in } \\
\text { dustrias) }\end{array}$ & $\begin{array}{c}D \\
\text { (Comercio) }\end{array}$ & $\begin{array}{c}\mathbf{E} \\
\text { (Servicios } \\
\text { y Transpor } \\
\text { tes) }\end{array}$ \\
\hline menos de 1.2 & 32 & - & 1 & 1 & - \\
\hline $1.2-2.6$ & 21 & 5 & 1 & 2 & 12 \\
\hline $2.6-4.0$ & - & 5 & 5 & 2 & 7 \\
\hline más de 4.0 & - & - & 1 & 1 & 4 \\
\hline Media de clase & 1.0543 & 2.5209 & 2.9616 & 3.2287 & 2.8590 \\
\hline Desviación standard & 0.5362 & 0.5130 & 1.2783 & 1.9554 & 0.8875 \\
\hline
\end{tabular}

Sin embargo, la media del índice es menor en los municipios de más de 50000 habitantes que en los de 30 a 50000 ; la diferencia no es significativa al $5 \%$, pero $-\mathrm{y}$ aquí una prueba de que lo pequeño de la muestra puede llevar a circunstancias extrañas-, si se elimina del intervalo de 30 a 50000 el municipio agrícola de San Luis Río Colorado, Sonora, con 42134 habitantes y bajo índice de urbanización (95.12), las medidas quedan como sigue:

Población municipal

$$
30000-50000
$$$$
\text { más de } 50000
$$

Indice medio de urbanización

3.0901

2.3070

que son significativamente diferentes al nivel de $2 \%$.

Éste es un resultado por demás interesante; puede ser accidental, por el tamaño pequeño de la muestra ( 23 municipios en los dos intervalos), pero combinado con los resultados antes comentados permite avanzar la conjetura de que la población municipal podría indicar diferencias de urbanización sólo si éstas son suficientemente grandes, o sea, hay una diferencia "crítica" de tamaños municipales por debajo de la cual el tamaño no indica diferencias de nivel de urbanización. Esta conjetura debe ser explorada, operacionalizada, probada y explicada en el futuro.

\section{El ÍNDice de URBanización y EL "desarrollo ZONAL" DE STERN}

En una etapa anterior de este proyecto, Stern [22], [23], clasificó las 111 zonas definidas por la Comisión Nacional de los Salarios Mínimos en siete "niveles de desarrollo". Los municipios analizados en el presente trabajo están localizados en cuarenta y siete de estas zonas. El cuadro 7 muestra la distribución conjunta del índice de urbanización y el nivel de desarrollo de Stern; aquí se invierte la terminología de Stern: el nivel 1 es el más bajo (corresponde al VII de Stern) y el 7 
Cuadro 7

DISTRIBUCTÓN CONJUNTA DEL ÍNDICE DE URBANIZACIÓN Y EL NIVEL ZONAL DE DESARROLLO DE STERN

\begin{tabular}{lccccccr}
\hline $\begin{array}{l}\text { Nivel de deB } \\
\text { In- arrollo }\end{array}$ & 1 & 2 & 3 & 4 & 5 & 6 & Total \\
$\begin{array}{l}\text { dice de } \\
\text { urbanización }\end{array}$ & (VII) \pm & (VI) & (V) & (IV) & (III) & (II) & \\
\hline Menor de 1.2 & - & 2 & 1 & 5 & 16 & 10 & 34 \\
$1.2-2.6$ & 3 & 4 & 5 & 6 & 17 & 6 & 41 \\
$2.6-4.0$ & 5 & 5 & 4 & 1 & 3 & 1 & 19 \\
Nayor a 4.0 & 1 & 3 & 1 & 1 & - & - & 6 \\
Sotal & 9 & 14 & 11 & 13 & 36 & 17 & 100 \\
\hline
\end{tabular}

\pm Los números romanos son los usados por stern.

el más alto. Los números de cada casilla corresponden al número de municipios cuyo índice de urbanización está en el intervalo correspondiente al renglón, localizados en una zona de nivel de desarrollo de Stern correspondiente a la columna. La estadística $\chi^{2}$ del cuadro 7 es significativa al $1 \%$, de donde el concepto de urbanización de este trabajo y el de desarrollo de Stern, el uno al nivel municipal y el otro al zonal, no son independientes. Sin embargo, la varianza del índice de urbanización entre niveles de Stern es sólo un $32.3 \%$ de la varianza total del índice entre municipios, de donde las diferencias del índice medio de urbanización entre niveles de desarrollo son significativas sólo al $5 \%$.

Se concluye que, sea por diferencias de construcción, sea por heterogeneidad zonal, el índice presentado en este trabajo y el índice que define los niveles de desarrollo de Stern, aunque interdependientes, parecen medir cosas distintas. Sería interesante obtener, en el futuro, índices de "urbanización" para las zonas de Stern, para dilucidar un poco esta conjetura.

\section{REFERENCIAS BIBLIOGRAFICAS}

[1] Bazzanella, W., "Industrialização e urbanização no Brasil", América Latina, 6 (1963), pp. 3-27.

[2] Carter, C. F., W. B. Reddaway y R. Stone, The Measurement of Production Movements. Cambridge University Press, 1948.

[3] Cinta, R., Aspectos socioeconómicos de la urbanización en México. Tesis profesional, Escuela Nacional de Ciencias Políticas y Sociales, UNAM, 1967.

[4] Clark, C., The Conditions of Economic Progress. Londres, MacMillan, 1940.

[5] Davis, K. y A. Casis, "Urbanization in Latin America", en P. K. Hatt y A. J. Reiss (compiladores), Cities and Society. Glencoe, The Free Press, 1961, pp. 141-156. 
[6] Davis, K. y H. Golden, "Urbanization and the Development of Pre-Industrial Areas", en P. K. Hatt y A. J. Reiss, op. cit., pp. 120-140.

[7] Dorselaer, J., "Las funciones socioeconómicas de las ciudades latinoamericanas", en L. Calderón, A. Calle y J. Dorselaer, Problemas de urbanización en América Latina. Estudios Sociológicos Núm. 13, FERES, Friburgo, 1963.

*[8] Dorselaer, J. y A. Gregory, La urbanización en América Latina, Vol. I. FERES, Friburgo, 1962.

[9] Fourastié, J., Le grand espoir du XXéme siècle. París, Presses Universitaires de France, 1952.

4 10] Germani, G., "Urbanización, secularización y desarrollo económico", Revista Mexicana de Sociología, 25 (1963), pp. 625-646.

[11] -, "El proceso de urbanización en Argentina". Naciones Unidas, Consejo Económico y Social, Santiago, Chile, 1959.

[12] Harbison, F. y C. Myers, Education, Manpower and Economic Growth. Nueva York, McGraw Hill, 1964.

[13] Hoselitz, B. S., Aspectos sociológicos del desarrollo económico. Barcelona, Editorial Hispano-Europea, 1962.

[14] Hotelling, H., "Analysis of a Complex of Statistical Variables into Principal Components", Journal of Educational Psychology, 24 (1933), 1? parte: pp. 417441 ; 2a parte: pp. 498-520.

[15] -, "Simplified Calculation of Principal Components", Psychometrica, 1 (1936), pp. 27-35.

[16] Kloek, T. y G. M. de Wit, "Best Linear and Best Linear Unbiased Index Numbers", Econometrica, 29 (1961), pp. 602-616.

[17] Kloek, T. y C. J. Van Rees, "On the Method of 'Deflated' Best Linear Index Numbers", Bulletin de l'Institut International de Statistique, 39 (1962), pp. 451-462.

[18] Redfield, R. y B. Singer, "The Cultural Role of the Cities", Economic Development and Cultural Change, 3 (1954).

[19] Reyna, J. L., M. Villa y K. Albrechtsen, "Dinámica de la estratificación social en algunas ciudades medianas y pequeñas de México", DEMoGraffa y ECoNOMfa, 1 (1967), pp. 368-394.

[20] Stavenhagen, R., Proyecto de investigación sobre la clase media mercantil en las pequeñas y medianas ciudades de México. Centro de Estudios Económicos y Demográficos, El Colegio de México, 1965. Mimeografiado.

[21] Steindl, J., Random Processes and the Growth of Firms. Londres, Griffin, 1965.

[22] Stern, C., Las regiones de México y sus niveles de desarrollo socioeconómico. Tesis profesional, Escuela Nacional de Ciencias Políticas y Sociales, UNAM, 1966.

[23]) -, "Un análisis regional de México", Demografía y Economfa, 1 (1967), pp. 92. 117.

[24] Theil, H., "Best Linear Index Numbers of Prices and Quantities", Econometrica, 28 (1960), pp. 464480.

[25] -, "Alternative Approaches to the Aggregation Problem", en P. Suppes, A. Tarski y E. Nagel (compiladores), Logic, Methodology, and Philosophy of Science. Stanford University Press, 1963, pp. 507-527.

[26] Tintner, G., "Some Applications of Multivariate Analysis to Economic Data", Journal of the American Statistical Association, 41 (1946), pp. 482-519.

[27] Véliz, C., Introducción, en C. Véliz (compilador), Obstacles to Change in Latin America. Oxford University Press, 1965, pp. 1-8.

[28] Weber, M., Economía y saciedad, México, Fondo de Cultura Económica, 1964, 2a edición.

[29] Wilkie, J., The Mexican Revolution: Federal Expenditure and Social Change since 1910. Berkeley, University of California Press, 1967.

[30] Wirth, L., "Urbanism as a Way of Life", en P. K. Hatt y A. J. Reiss, op. cit., pp. $46-63$. 